\title{
Reconstruction of gene networks associated with autism and related to mTOR signaling pathway using ANDSystem
}

\author{
O.V. Saik*, E.A. Trifonova, T.M. Khlebodarova, V.A. Ivanisenko \\ Institute of Cytology and Genetics SB RAS, Novosibirsk, Russia \\ *e-mail:saik@bionet.nsc.ru
}

Key words: autism spectrum disorder (ASD), ANDSystem, associative gene networks, mTOR pathway

Motivation and Aim: According to epidemiological studies it is known, that autism spectrum disorder (ASD) can affect near 1\% of the world's population. The symptoms of ASD includes the presence of limited interests, problems in social communication, stereotyped and repetitive behavior. It is postulated that one of the mechanisms involved in ASD pathogenesis is disruption in mTOR signaling pathway [1]. The aim of this work was reconstruction and analysis of gene networks associated with ASD, related to the mTOR signaling pathway, by ANDSystem [2] in order to identify potential gene-targets that are promising for drug development studies.

Methods and Algorithms: The list of genes associated with ASD was created based on the information from Malacards database (https:/www.malacards.org/) and ANDSystem [2]. Genes involved in mTOR signaling pathway were taken from KEGG database. Reconstruction and analysis of gene networks was performed using ANDSystem. Gene Ontology enrichment analysis was made by DAVID 6.8 .

Results: Analysis of information from Malacards and ANDSystem revealed 452 genes associated with ASD. The reconstructed gene network contains 12147 interactions between these proteins and genes. There were 271 expression regulation relations in the reconstructed gene network. According to KEGG database 151 genes are involved in mTOR signaling pathway and it was found that eight (EIF4E, HRAS, IGF1, PRKCB, PTEN, TSC1, TSC2, WNT2) are related with ASD. Among them PTEN had the highest value of betweenness centrality (16214) and it was in the top 20 most central genes of ASD gene network. Using ANDSystem it was found that 82 genes/proteins from 151 involved in mTOR signaling pathway were directly linked with 193 genes/proteins associated with ASD. This 193 genes/proteins associated with ASD and interacting with mTOR signaling pathway were enriched with the following Gene Ontology biological processes: positive regulation of $\mathrm{B}$ cell proliferation, antigen processing and presentation, immune response, response to drug, protein localization to synapse.

Conclusion: The results obtained in this work suggest that genes/proteins from the mTOR signaling pathway have central role in the gene network associated with ASD. The mTOR signaling pathway is highly connected to ASD gene network. Among them PTEN could be the most perspective for further investigation as potential drug target. Acknowledgements: Reconstruction of autism gene network was supported by Project Fundamental Research of SB RAS No. 0324-2018-0021. Reconstruction of gene network of mTOR signaling pathway was supported by the RFBR grant 18-41-540004.

\section{References}

1. Trifonova E.A. et al. (2017) Molecular mechanisms of autism as a form of synaptic dysfunction. Russian Journal Genetics: Applied Research. 7(8):869-877.

2. Ivanisenko V.A. et al. (2015) ANDSystem: an Associative Network Discovery System for automated literature mining in the field of biology. BMC Syst Biol. 9(Suppl 2):S2. 